H. Omoto and Y. Kimura

Nagoya Math. J.

Vol. 125 (1991), 15-31

\title{
ON $\varepsilon$-APPROXIMATE SINGULARITIES OF AUTONOMOUS SYSTEMS OF VORTEX TYPE
}

\author{
HIDEO OMOTO AND YOSHIFUMI KIMURA
}

\section{§ 0. Introduction}

Let us consider three vortex-filaments $z_{j}(t)$ with strength $\Gamma_{j}(j=$ $1,2,3)$ in the complex plane $\mathbf{C}$. Then the system of motion equations is given by

$$
\frac{d z_{j}}{d t}=\sqrt{-1} \sum_{\substack{k=1 \\(k \neq j)}} \frac{\Gamma_{k}}{\bar{z}_{j}-\bar{z}_{k}} \quad(j=1,2,3) .
$$

This system (E) is defined on $V=\mathbf{C}^{3}-\Delta$, where $\Delta=\left\{\left(z_{1}, z_{2}, z_{3}\right) \in \mathbf{C}^{3} ; z_{j}=z_{k}\right.$ for $j \neq k\}$ is the super-diagonal set of $\mathbf{C}^{3}$. Let $\operatorname{Sol}(\mathrm{E})$ be the space of all smooth solutions of $(\mathrm{E})$ and let $\psi: V \rightarrow \operatorname{Sol}(E)$ be a smooth map defined as follows: For any $\alpha=\left(\alpha_{1}, \alpha_{2}, \alpha_{3}\right) \in V, \psi(\alpha)$ is the solution with initial values $\alpha$.

It is well-known (cf. [2], p. 260) that if three points $\alpha_{j}$ of $\alpha=\left(\alpha_{1}, \alpha_{2}, \alpha_{3}\right)$ make a regular triangle in $\mathbf{C}$, then $\psi(\alpha)$ becomes a rotational motion about these center of mass, which is called rigid-rotation. This solution $\psi(\alpha)$ has no singular points (cf. Definition 2.1). Now instead of $\alpha$, let us take $\alpha(\varepsilon)=\alpha+\varepsilon \beta$ as initial values, where $\varepsilon$ is a small parameter and $\beta \in \mathbf{C}^{3}$. Then using computers, we find that $\psi(\alpha(\varepsilon))$ has a singular point at a time $t=T_{0}(\varepsilon)$, and that $T_{0}(\varepsilon)$ seems to approach asymptotically to a $\log (1 / \varepsilon)+b$ as $\varepsilon \rightarrow 0$, for constants $a, b$ (see Figure). We may set the following problems:

(A) Is it true that $T_{0}(\varepsilon) \sim a \log (1 / \varepsilon)+b(\varepsilon \rightarrow 0)$ ?

(B) If (A) is correct, explain how the above constants $a$ and $b$ are determined from the given differential equations (E).

It doesn't seem that such problems have been treated yet.

In this paper we generalize the motion equations $(\mathrm{E})$ on $\mathbf{C}$ to autonomous systems of vortex type on $\mathbf{C}^{n}$ defined in $\S 1$. We can also consider

Received January 22, 1991. 


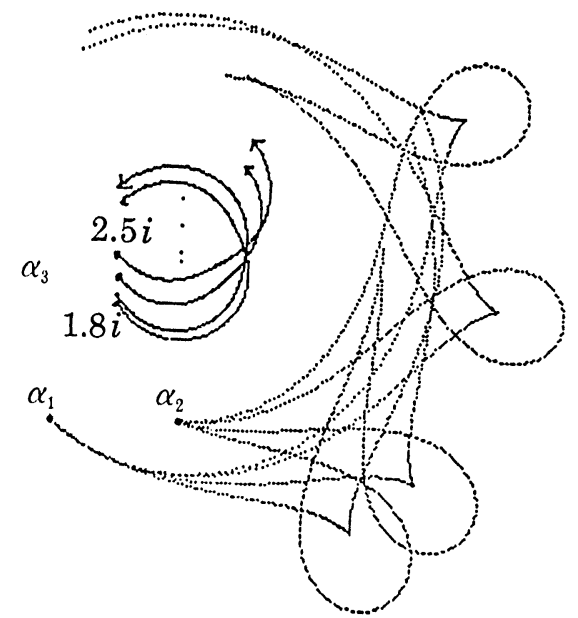

Figure. Integral curves of (E) with initial values $\alpha_{1}=-1, \alpha_{2}=1$ and (1) $\alpha_{3}=2.5 i$; (2) $\alpha_{3}=2.2 i$; (3) $\alpha_{3}=1.9 i$; (4) $\alpha_{3}=1.8 i$. where $i=\sqrt{-1} \Gamma_{1}=-2, \Gamma_{2}=1, \Gamma_{3}=4$.

the same problems with respect to $\varepsilon$-approximation of such autonomous systems defined in $\S 2$. Then we prove Theorem 3.6 in $\S 3$ which solves partially our problems.

In preparing this paper we have received useful advices and sincere encouragements from Professor Y. Shikata. We would like to express our cordial gratitude to him.

\section{§1. Vortex-Hamiltonian structures}

1.1. Notation. Let $\mathbf{C}^{m}$ be the space of $m$ complex variables $\boldsymbol{z}_{0}^{1}, \boldsymbol{z}_{0}^{2}$, $\cdots, z_{0}^{m}$. The elements of $\mathbf{C}^{m}$ are written as vectors of length $m$. We put $z_{0}=\left(z_{0}^{1}, \cdots, z_{0}^{m}\right)$ and

$$
\left\{\begin{array}{l}
\bar{z}_{0} d z_{0}=\sum_{\alpha=1}^{m} \bar{z}_{0}^{\alpha} d z_{0}^{\alpha}, \\
d z_{0} \wedge d \bar{z}_{0}=\sum_{\alpha=1}^{m} d z_{0}^{\alpha} \wedge d \bar{z}_{0}^{\alpha} .
\end{array}\right.
$$

For any $\mathbf{C}^{\infty}$-complex valued function $f$ on $\mathbf{C}^{m}$, we define the vector-valued function $\partial f / \partial z_{0}$ by

$$
\frac{\partial f}{\partial z_{0}}=\left(\frac{\partial f}{\partial z_{0}^{1}}, \frac{\partial f}{\partial z_{0}^{2}}, \cdots, \frac{\partial f}{\partial z_{0}^{m}}\right)
$$

and for any smooth vector-valued function $X=\left(X^{1}, X^{2}, \cdots, X^{m}\right)$ on $\mathbf{C}^{m}$, the $m \times m$-matrix $\partial X / \partial z_{0}$ associated with to the function $X$ is defined by 


$$
\frac{\partial X}{\partial z_{0}}=\left(\begin{array}{c}
\frac{\partial X^{1}}{\partial z_{0}^{1}}, \cdots, \frac{\partial X^{1}}{\partial z_{0}^{m}} \\
\cdots \cdots \cdots \cdot \\
\frac{\partial X^{m}}{\partial z_{0}^{1}}, \cdots, \frac{\partial X^{m}}{\partial z_{0}^{m}}
\end{array}\right) .
$$

1.2. Let us set $V_{0}=\mathbf{C}^{m}$. We shall now consider motions of $n$-points $z_{j}(t)(j=1, \cdots, n)$ in $V_{0}$. First one notices that there is the canonical Kaehler form $\Omega_{0}$ on $V_{0}$, defined by

$$
\Omega_{0}=\sqrt{-1} d z_{0} \wedge d \bar{z}_{0}
$$

and that putting

$$
\theta_{0}=\frac{\sqrt{-1}}{2}\left(z_{0} d \bar{z}-\bar{z}_{0} d z_{0}\right)
$$

it follows that $\theta_{0}$ is a real 1 -form on $V_{0}$ such that

$$
d \theta_{0}=\Omega_{0} .
$$

Set $V_{j}=\mathbf{C}^{m},(j=1, \cdots, n)$ and let $V=V_{1} \times \cdots \times V_{n}$. For each $j$, let $\pi_{j}$ be the $j$-th projection of $V$ onto $V_{0}$, defined by

$$
\pi_{j}\left(z_{1}, \cdots, z_{n}\right)=z_{j} \quad \text { for }\left(z_{1}, \cdots, z_{n}\right) \in V .
$$

Definition 1.1. Let $\Gamma_{1}, \cdots, \Gamma_{n}$ be non-zero real constants and put

$$
\theta_{j}=\pi_{j}\left(\theta_{0}\right), \quad(j=1, \cdots, n) .
$$

Then

$$
\theta=\sum_{j=1}^{n} \Gamma_{j} \theta_{j}
$$

is called the fundamental form with strength $\Gamma_{1}, \cdots, \Gamma_{n}$ on $V$. Further

$$
\Omega=d \theta
$$

is a non-degenerate closed 2-form on $V$, and so we call $(V, \Omega)$ the symplectic manifold with strength $\Gamma_{1}, \cdots, \Gamma_{n}$.

Let $(V, \Omega)$ be a symplectic manifold as in the above definition. We can define the action of the general linear group $G L(m, \mathbf{C})$ and the additive group $\mathbf{C}^{m}$ on this space $V$ as follows: For all $g \in G L(m, \mathbf{C})$ and $\alpha \in \mathbf{C}^{m}$,

(i ) $g\left(z_{1}, \cdots, z_{n}\right)=\left(g z_{1}, \cdots, g z_{n}\right)$,

(ii) $\alpha\left(z_{1}, \cdots, z_{n}\right)=\left(\alpha+z_{1}, \cdots, \alpha+z_{n}\right)$ 
for any $\left(z_{1}, \cdots, z_{n}\right) \in V$.

In particular $\mathbf{C}^{*}=\mathbf{C}-\{0\}$ being regarded as the diagonal subgroup of $G L(m, \mathbf{C}), V$ admits $\mathbf{C}^{*}$-actions. We denote by $U(m)$ the unitary group which acts on $V$.

Now let $\Delta$ be a closed subset of $V$ with the following properties: $\Delta$ is invariant under the groups $U(m), \mathbf{C}^{*}$ and $\mathbf{C}^{m}$ respectively, and each projection $\pi_{j}: \tilde{V}=V-\Delta \rightarrow V_{j}$ is onto for $j=1, \cdots, n$. $\tilde{V}$ is also invariant under these groups. Here instead of $(V, \Omega)$ we take this open symplectic submanifold $(\tilde{V}, \Omega)$ of $\tilde{V}$. Finally let $H: \tilde{V} \rightarrow R$ be a smooth function (called Hamiltonian function), satisfying the following three conditions:

(a) $U(m)$ and $\mathbf{C}^{m}$-invariant.

(b) $\mathbf{C}^{*}$-semiinvariant, that is, for any $a \in \mathbf{C}^{*}$ and $\left(z_{1}, \cdots, z_{n}\right) \in \tilde{V}$,

$H\left(a z_{1}, \cdots, a z_{n}, \bar{a} \bar{z}_{1}, \cdots, \bar{a} \bar{z}_{n}\right)=H\left(z_{1}, \cdots, z_{n}, \bar{z}_{1}, \cdots, \bar{z}_{n}\right)+\gamma \log |a|^{2}$, where $\gamma$ is a real constant independent of $a$ and $\left(z_{1}, \cdots, z_{n}\right)$.

(c) $\partial \tilde{\partial} H=0$,

where $\partial$ and $\bar{\partial}$ mean the derivations of type $(1,0)$ and $(0,1)$, respectively.

Thus the triplet $(\tilde{V}, \Omega, H)$ is called Hamiltonian structure of vortex type.

Definition 1.2. Let $(\tilde{V}, \Omega, H)$ be as above. A real smooth vector field $\tilde{X}$ is called of vortex type if

$$
\tilde{X}\lrcorner \Omega=-d H .
$$

Let $\tilde{X}$ be of vortex type. We express this vector field $\tilde{X}$, using vectorvalued coordinates $z_{1}, \cdots, z_{n}$ of $V . \tilde{X}$ can be written as

$$
\tilde{X}=\sum_{j=1}^{n} \bar{X}_{j}(z, \bar{z}) \partial / \partial z_{j}+\sum_{j=1}^{n} X_{j}(z, z) \partial / \partial \bar{z}_{j},
$$

where for each $j, z_{j}=\left(z_{j}^{1}, \cdots, z_{j}^{m}\right)$ and $\bar{X}_{j}$ is the complex conjugate $X_{j}$ and $\bar{X}_{j} \partial / \partial z_{j}$ stands for $\sum_{\alpha=1}^{m} \bar{X}_{j}^{\alpha} \partial / \partial z_{j}^{\alpha}$.

Then we find from (1.5)

$$
\bar{X}_{j}=-\sqrt{-1} \frac{1}{\Gamma_{j}} \frac{\partial H}{\partial \bar{z}_{j}}
$$

and

$$
X_{j}=\sqrt{-1} \frac{1}{\Gamma_{j}} \frac{\partial H}{\partial z_{j}} .
$$


Moreover in terms of the condition (c) for $H$, it follows that the $\bar{X}_{j}$ are anti-holomorphic vector-valued functions on $\tilde{V}$. Therefore integral curves $z(t)=\left(z_{1}(t), \cdots, z_{n}(t)\right)$ of $\tilde{X}$ satisfy the following system of differential equations, called an autonomous system of vortex type

$$
\frac{d z_{j}}{d t}=X_{j}\left(z_{1}, \cdots, z_{n}\right), \quad(j=1, \cdots, n) .
$$

\section{$\S 2$. Singularities and properties of autonomous systems of vortex type}

We use the same notations as before.

Definition 2.1. Let $z(t)=\left(z_{1}(t), \cdots, z_{n}(t)\right)$ be a solution of (1.7) and let $\pi_{j}: \tilde{V} \rightarrow \mathbf{C}^{m}$ be the $j$-th projection as in 1.2 for $j=1, \cdots, n$. This solution $z(t)$ is singular, more precisely $j$-singular, at a time $t=t_{0}$ if there exists an index $j$ such that the image curve of $z_{j}(t)=\pi_{j}(z(t))$ in $\mathbf{C}^{m}$ has a vanishing derivative at $t=t_{0}$, that is

$$
\left.\frac{d z_{j}}{d t}\right|_{t=t_{0}}=0
$$

Now we assume that there exists a non-singular solution $z(t)$ of (1.7) with initial values $\alpha=\left(\alpha_{1}, \cdots, \alpha_{n}\right) \in \tilde{V}$ at $t=0$. Let $z(t ; \varepsilon)$ be the solution with initial values $z(0 ; \varepsilon)=\alpha+\varepsilon \beta$ for a small $|\varepsilon|>0$. Put

$$
w(t)=\left.\frac{d}{d \varepsilon} z(t ; \varepsilon)\right|_{\varepsilon-0},
$$

and

$$
\tilde{z}(t ; \varepsilon)=z(t)+\varepsilon w(t)
$$

which we call the $\varepsilon$-order approximation of $z(t ; \varepsilon)$.

We now want to obtain a value $t_{0}$ of $t$ such that for some $k$,

$$
\frac{d \tilde{z}_{k}}{d t}\left(t_{0} ; \varepsilon\right)=0
$$

For this purpose we write down a system of differential equations which the above unknown vector-valued function $w(t)$ satisfies. Set

$$
\bar{X}=\left(\bar{X}_{1}, \cdots, \bar{X}_{n}\right)
$$

where the $\bar{X}_{j}$ are defined by (1.6), then $d z(t ; \varepsilon) / d t=\bar{X}(z(t ; \varepsilon))$. By differentiation in $\varepsilon$, 


$$
\frac{d w_{j}(t)}{d t}=\sum_{j=1}^{n} \frac{\partial \bar{X}_{j}}{\partial \bar{z}_{j}} \bar{w}_{j}(t) \quad(j=1, \cdots, n),
$$

or in the matrix form,

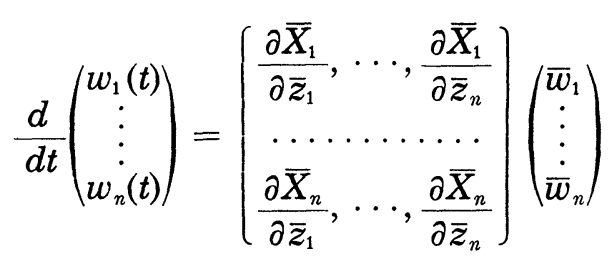

which is the system of differential equations for the $w$ 's. Here one notes that the $\partial \bar{X}_{j} / \partial \bar{z}_{k}$ are $m \times m$-matrices. For convenience sake, let us put

$$
\left\{\begin{array}{l}
\bar{A}_{i j}(t)=\frac{\partial \bar{X}_{j}}{\partial \bar{z}_{j}}(t) \quad(1 \leqq i, j \leqq n), \\
\bar{A}(z)=\left(\begin{array}{c}
\bar{A}_{11}(z), \cdots, \bar{A}_{1 n}(z) \\
\cdots \cdots \cdots, \cdots \\
\bar{A}_{n 1}(z), \cdots, \bar{A}_{n n}(z)
\end{array}\right) .
\end{array}\right.
$$

Then $\left(2.2^{\prime}\right)$ can be written as follows;

$$
\frac{d w(t)}{d t}=A(z(t)) \bar{w}(t)
$$

where $w(t)={ }^{t}\left(w_{1}(t), \cdots, w_{n}(t)\right)$. Putting $z(0 ; \varepsilon)=\alpha+\varepsilon \beta$. We find that $w(t)$ is a solution of (2.4) with $w(0)=\beta$. From the above discussions our problem is summarized as follows: Let $z(t)$ be a non-singular solution of (1.7) with $z(0)=\alpha$ and $w(t)$ a solution of (2.4) such that $w(0)=\beta$. Then the problem is to find a value $t_{0}$ of $t$ satisfying the following equation: For some index $k$.

$$
\frac{d \tilde{z}_{k}}{d t}(t)+\varepsilon \sum_{j=1}^{n} \bar{A}_{k j}(z(t)) \bar{w}_{j}(t)=0
$$

We shall solve this problem in case where the above solution $z(t)$ is $U(m)$ - or $\mathbf{C}^{*}$-solution defined in $\S 3$.

2.2. In this paragraph we examine some properties of the vector field $X$ and the matrix $\bar{A}(z)$ which are defined in 2.1. First of all we obtain the following

Lemma 2.2. For $g \in U(m)$ and $a \in \mathbf{C}^{*}$,

$$
\bar{X}(g \alpha)=g \bar{X}(\alpha)
$$


and

$$
\bar{X}(a \alpha)=\frac{1}{\bar{a}} \bar{X}(\alpha)
$$

Proof. Since the Hamiltonian $H(z, \bar{z})$ is $U(m)$-invariant, for any $g=$ $\left(g_{a b}\right) \in U(m)$ and $\alpha \in \tilde{V}$, we get

$$
\text { (*) } \quad \sum_{b=1}^{m} \bar{g}_{a b} \frac{\partial H}{\partial \bar{z}_{j}^{b}}(g \alpha)=\frac{\partial H}{\partial \bar{z}_{j}^{a}}(\alpha), \quad(j=1, \cdots, n)
$$

for $z_{j}=\left(\boldsymbol{z}_{j}^{1}, \cdots, \boldsymbol{z}_{j}^{m}\right)$.

Using matrix notations, $(*)$ are expressed as

$$
{ }^{t} \bar{g} \frac{\partial H}{\partial \bar{z}_{j}}(g \alpha)=\frac{\partial H}{\partial \bar{z}_{j}}(\alpha), \text { for all } j .
$$

Therefore from Definition (1.6) of the $\bar{X}_{j}$, it follows

$$
\bar{X}_{j}(g \alpha)={ }^{t} \overline{\boldsymbol{g}}^{-1} X_{j}(\alpha), \quad(j=1, \cdots, n) .
$$

As $g$ is unitary, we have (2.6).

Since $H$ is $\mathbf{C}^{*}$-semiinvariant, (2.8) is also satisfied for $a \in \mathbf{C}^{*}$, and so (2.7) is proved.

Q.E.D.

From this lemma and Definition (2.3) of the matrices $\bar{A}_{i j}$ and $\bar{A}$ we can prove immediately the following

Proposition 2.3. For $g \in U(m)$ and $a \in \mathbf{C}^{*}$,

$$
\bar{A}_{i j}(g \alpha)=g A_{i j}(\alpha) \bar{g}^{-1},
$$

i.e.,

$$
\bar{A}(g \alpha)=g \bar{A}(\alpha) \bar{g}^{-1},
$$

and

$$
\bar{A}(a \alpha)=\frac{1}{\bar{a}^{2}} A(\alpha) \quad \text { for any } \alpha \in \tilde{V}
$$

Finally we obtain the following proposition which states the so-called angular momentum invariance.

Proposition 2.4. We have

$$
\sum_{j=1}^{n} \Gamma_{j} \bar{X}_{j}=0
$$


and

$$
\sum_{j=1}^{n} \Gamma_{j} \bar{z}_{j} \bar{X}_{j}=-\sqrt{-1} \gamma,
$$

where $\Gamma_{j}$ is the strength of the $j$-th point $z_{j}(j=1, \cdots, n)$ and $\gamma$ is the constant defined in (c) of 1.2 .

Proof. From $\mathbf{C}^{m}$-invariance of $H$ we get

$$
\left.\frac{\partial H(z+a, \bar{z}+\bar{a})}{\partial \bar{a}^{\alpha}}\right|_{a=0}=\sum_{j=1}^{n} \frac{\partial H}{\partial \bar{z}_{j}^{\alpha}}=0
$$

for $a=\left(a^{1}, \cdots, a^{n}\right)$ and $\alpha=1, \cdots, m$. Therefore from (1.6) we have

$$
\sum_{j=1}^{n} \Gamma_{j} \bar{X}_{j}(z)=0
$$

which shows (2.11).

(2.12) can be proved, using

$$
\left.\frac{\partial H(a z, \bar{a} \bar{z})}{\partial \bar{a}}\right|_{a=1}=\sum_{j=1}^{n} \frac{\partial H}{\partial \bar{z}_{j}} \bar{z}_{j}=\gamma \quad \text { for } a \in \mathbf{C}^{*} .
$$

Q.E.D.

In virtue of (2.11) we have the following

Corollary 2.5. The determinant $|A|$ of $A$ is zero i.e.,

$$
|A|=0 \text {. }
$$

\section{$\S 3$. The kinds of solutions}

\subsection{Rigid rotational solutions}

3.1.1. We start from the following

Definition 3.1. A solution $z(t)$ of (1.7) is called a rigid rotational solution or $U(m)$-solution with initial values $\alpha=\left(\alpha_{1}, \cdots, \alpha_{n}\right)$ at $t=0$, if there exists a 1-parameter group $S: R \rightarrow U(m)$, that is,

$$
S(t)=\exp t C \quad \text { for all } t \in R
$$

such that

$$
z(t)=S(t) \alpha,
$$

where $C$ denotes an anti-hermitian matrix such that $C \alpha_{j} \neq 0$. 
Let $z(t)$ be a $U(m)$-solution defined by (3.1). Then

$$
\dot{S} \alpha=\bar{X}(S \alpha)
$$

where $\dot{S}=d S / d t$. It follows from (2.6) and $C=S^{-1} \dot{S}$

$$
C \alpha=\bar{X}(\alpha) \text {. }
$$

Furthermore differentiating $S(t)^{-1} \bar{X}(S(t) \alpha)=C \alpha$ with respect to $t$, we find

$$
\bar{A}(\alpha) \bar{C} \bar{\alpha}=C^{2} \alpha .
$$

Now let $\tilde{z}(t ; \varepsilon)=z(t)+\varepsilon w(t)$ be an $\varepsilon$-order approximation such that $\tilde{z}(0 ; \varepsilon)=\alpha+\varepsilon \beta$ as explained in $\S 2$. Then $w(t)$ satisfies

$$
\frac{d w(t)}{d t}=S(t) \bar{A}(\alpha) \bar{S}(t)^{-1} \bar{w}(t),
$$

because of (2.4).

Let us set

$$
v(t)=S(t)^{-1} w(t) .
$$

Then the system of linear differential equations for $v(t)$ equivalent to (3.4) is

$$
\frac{d v(t)}{d t}=\bar{A}(\alpha) \bar{v}(t)-C v(t) .
$$

We introduce an $R$-linear map $B: V \rightarrow V$ defined by

$$
B(\xi)=-C \xi+\bar{A}(\alpha) \bar{\xi}, \quad \xi \in V .
$$

Using this map $B$, (3.6) is expressed in the form

$$
\frac{d v}{d t}=B(v) .
$$

In order to solve (3.8), it is convenient to write down (3.8) in real forms. We identify $V$ with $V_{R}=R^{m n} \times R^{m n}$ by the map $\phi$ defined as follows: Let $\xi=x+\sqrt{-1} y \in V$ for $x$ and $y$ real. Then

$$
\phi(\xi)=(x, y) \in V_{R} .
$$

For simplicity we denote $\phi(\xi)=\hat{\xi}$. Let $\hat{v}(t)=\left(v_{1}, v_{2}\right) \in V_{R}, C=C_{1}+\sqrt{-1} C_{2}$, and $A(\alpha)=A_{1}+\sqrt{-1} A_{2}$. Then (3.8) is written in the space $V_{R}$ as follows;

$$
\frac{d}{d t}\left(\begin{array}{l}
v_{1} \\
v_{2}
\end{array}\right)=\hat{B}\left(\begin{array}{l}
v_{1} \\
v_{2}
\end{array}\right),
$$


where

$$
\hat{B}=\left(\begin{array}{r}
A_{1}-C_{1},-A_{2}+C_{2} \\
-A_{2}-C_{2},-A_{1}+C_{1}
\end{array}\right)
$$

If $B(\xi)=\lambda \xi$ for some vector $\xi \in V$ and a real number $\lambda$, then $\hat{\xi}=\phi(\xi)$ is an eigenvector of $\hat{B}$ corresponding to $\lambda$. As a consequence of it, we obtain the following

Proposition 3.1. $B$ has the eigenvalue 0 and the vector $C \alpha$ is the 0-eigenvector.

Proof. From Definition (3.7) of $B$ and (3.3) we have

$$
B(C \alpha)=-C^{2} \alpha+\bar{A}(\alpha) \bar{C} \bar{\alpha}=0 .
$$

But $C \alpha \neq 0$ from the assumption, which implies this proposition. Q.E.D.

Moreover we can show by direct calculations the following

Lemma 3.2. Let us assume that

$$
C A(\alpha)=A(\alpha) C .
$$

Then the characteristic equation of $\hat{B}$ is

$$
|(\lambda E+\bar{C})(\lambda E+C)-A \bar{A}|=0,
$$

where $E$ is the unit matrix.

In particular in case of $m=1$ we get following

Corollary 3.3. The matrix $\hat{B}$ has eigenvalues $0,-c$, and $-\bar{c}$. And 0 is of multiplicity $\geqq 2$, where $C$ reduces to the scalor matrix (c).

Proof. As $m=1$, the condition (3.10) is automatically fulfiled. From (3.11) and Corollary 2.5, $-c$ and $-\bar{c}$ are eigenvalues of $\hat{B}$. On the other hand, (3.11) reduces to $\left|\left(\lambda^{2}+c \bar{c}\right) E-A \bar{A}\right|=0$, whence the multiplicity of eigenvalue 0 is not less than $2 . \quad$ Q.E.D.

3.1.2. Now let us return to the discussions of singularities. Let $\lambda_{1}, \cdots, \lambda_{2}$ be eigenvalues of $\hat{B}$ and let $m_{j}$ be the multiplicity of $\lambda_{j},(j=1$, $\cdots, l)$. We denote by $\hat{W}\left(\lambda_{j}\right)$ the eigenspace associated with $\lambda_{j}$ of multiplicity $m_{j}$;

$$
\hat{W}\left(\lambda_{j}\right)=\left\{\hat{\xi} \in V_{R} ;\left(\lambda_{j}-\hat{B}\right)^{m_{j}} \hat{\xi}=0\right\} .
$$

Remember $v(t)$ is the solution of (3.8) with $v(0)=\beta$ for $\beta=x+\sqrt{-1} y \in V$. 
Since $V_{R} \otimes C$ is decomposed into the direct sum of $\hat{W}\left(\lambda_{1}\right), \cdots, \hat{W}\left(\lambda_{l}\right)$. $\hat{\beta}=(x, y) \in V_{R}$ is expressed as a sum of $\hat{W}\left(\lambda_{j}\right)$-components of $\hat{\beta}$. We say that $\lambda_{j}$ is associated with $\beta$, if the $\hat{W}\left(\lambda_{j}\right)$-component is not zero.

Definition 3.4. Let $\lambda_{j}$ be an eigenvalue of $\hat{B}$ associated with $\beta$. $\lambda_{j}$ is called dominant for $\beta$, when

(i) $\operatorname{Re}\left(\lambda_{j}\right)>0$,

(ii) $\operatorname{Re}\left(\lambda_{j}\right)$ is greater than the real part of any other eigenvalue associated with $\beta$, where $\operatorname{Re}(\lambda)$ means the real part of $\lambda$.

In order to express the solution $v(t)$ of (3.8), using eigenvalues and eigenvectors of $\hat{B}$, we shall introduce the following notations: Let $\lambda$ be an eigenvalue of $\hat{B}$ and let $\hat{\beta}_{0} \in \hat{W}(\lambda)$. If $\lambda$ is real, we may assume that $\hat{\beta}_{0}$ is a real vector. At first in case where $\lambda$ is real, we can write $\hat{\beta}_{0}, \beta_{0}$ in the forms

$$
\hat{\beta}_{0}=(x, y) \in V_{R} \quad \text { and } \quad \beta_{0}=x+\sqrt{-1} y \in V .
$$

With these notations let $\beta_{1}, \cdots, \beta_{k} \in \hat{W}(\lambda)$, and

$$
\text { (I) } P(t)=c_{1} \beta_{1}+t c_{2} \beta_{2}+\cdots+t^{k-1} c_{k} \beta_{k} \text {. }
$$

On the other hand if $\lambda=a+\sqrt{-1} b$ is imaginary, we may write

$$
\hat{\beta}_{0}=\hat{\beta}_{1}+\sqrt{-1} \hat{\beta}_{2} \in V_{R} \otimes C
$$

for $\hat{\beta}_{j}=\left(x_{\jmath}, y_{j}\right) \in V_{R},(j=1,2)$. Let

$$
\beta_{j}=x_{j}+\sqrt{-1} y_{j} \in V, \quad(j=1,2)
$$

and put for any real number $c_{j}(j=1,2)$,

$$
\left[\hat{\beta}_{0}: c_{1}, c_{2}\right]=c_{1}\left(\cos b t \cdot \beta_{1}-\sin b t \cdot \beta_{2}\right)+c_{2}\left(\sin b t \cdot \beta_{1}+\cos b t \cdot \beta_{2}\right),
$$

for $a=\operatorname{Re}(\lambda)$ and $b=\operatorname{Im}(\lambda)$. Further for any $\hat{\beta}_{1}, \cdots, \hat{\beta}_{k} \in \hat{W}(\lambda)$, we set

$$
\text { (II) } P(t)=\left[\hat{\beta}_{1}: c_{11}, c_{12}\right]+t\left[\hat{\beta}_{2}: c_{21}, c_{22}\right]+\cdots+t^{k-1}\left[\hat{\beta}_{k}: c_{k 1}, c_{k 2}\right] \text {. }
$$

We call the above functions $P(t)$ defined by (I), (II) for an eigenvalue $\lambda$, $\hat{W}(\lambda)$-polynomial functions of degree $k-1$. With these notations we can express the solution $v(t)$ of (3.8) with initial values $\beta$. Let $\left\{\lambda_{1}, \cdots, \lambda_{s}\right.$, $\left.\bar{\lambda}_{1}, \cdots, \bar{\lambda}_{s}, \cdots, \lambda_{s+1}, \cdots, \lambda_{r}\right\}$ be all eigenvalues associated with $\beta$, where $\bar{\lambda}_{j}$ is complex-conjugate to $\lambda_{j},(j=1, \cdots, s)$ and $\lambda_{s+1}, \cdots, \lambda_{r}$ are real. Then from the well-known theorem of differential equations with constant coefficients (cf. [3]) it follows 


$$
v(t)=\sum_{j=1}^{r} e^{a_{j} t} P_{j}(t)
$$

where $\lambda_{j}=a_{j}+\sqrt{-1} b_{j}$ and $P_{j}(t)$ are $\hat{W}\left(\lambda_{j}\right)$-polynomial functions.

Remark. Let all notations be as above. Let $\hat{\beta}=\sum_{j=1}^{s} \hat{\beta}_{j}+\sum_{j=1}^{s} \hat{\beta}_{j}$ $+\sum_{k=s+1}^{r} \hat{\beta}_{k}$. If $\hat{\beta}_{j}$ is an eigenvector, that is, $\hat{B}_{\hat{\beta}}=\lambda_{j} \hat{\beta}_{j}$, then $P_{j}(t)$ is of degree 0 . Therefore for the $\varepsilon$-order approximation $\tilde{z}(t ; \varepsilon)=z(t)+\varepsilon S(t) v(t)$, we have from (3.12) and $z(t)=S(t) \alpha$,

$$
S(t)^{-1} \frac{d \tilde{z}}{d t}=C \alpha+\varepsilon \sum_{j=1}^{r} e^{a_{j t}} \bar{A}(\alpha) \bar{P}_{j}(t) .
$$

Here we need the following.

Definition 3.5. An eigenvalue $\lambda$ of $\hat{B}$ is simply dominant for $\beta$ if $\lambda$ is dominant (cf. Definition 3.4) and if the $\hat{W}(\lambda)$-component of $\beta$ is the eigenvector for $\lambda$.

Let us suppose that the above eigenvalue $\lambda_{r}$ is simply dominant for $\beta$. Then from the preceding remark

$$
P(t)=\beta_{r},
$$

where $\hat{\beta}_{r}$ is the $\hat{W}\left(\lambda_{r}\right)$-component of $\hat{\beta}$.

Moreover we introduce a linear map $\bar{A}_{k}(\alpha): V \rightarrow V_{k}=C^{m}(k=1, \cdots, n)$ defined by

$$
\bar{A}_{k}(\alpha) \beta_{0}=\sum_{j=1}^{n} \bar{A}_{k j}(\alpha) \beta_{0 j}
$$

for any $\beta_{0}=\left(\beta_{01}, \cdots, \beta_{0 n}\right) \in V$. Finally we assume that for some index $k$, there exists a non-zero real number $\delta_{k}$ such that

$$
C \alpha_{k}=\delta_{k} \bar{A}(\alpha) \bar{\beta}_{r},
$$

where $\alpha=\left(\alpha_{1}, \cdots, \alpha_{n}\right) \in \tilde{V}$.

We say that the vector $\beta$ satisfying (3.15) is $k$-dominant parallel to $\alpha$ with a ratio-constant $\delta_{k}$. Under the condition (3.15) for $\beta$, we have from

$$
\frac{d \tilde{z}_{k}(t ; \varepsilon)}{d t}=S(t) \bar{A}_{k}(\alpha)\left\{\delta_{k} \beta_{r}+\varepsilon e^{\lambda_{r} t}\left[\bar{\beta}_{r}+\sum_{j=1}^{r-1} e^{\left(a_{j}-\lambda_{r}\right) t} \bar{P}_{j}(t)\right]\right\}
$$

Let $t=T(\varepsilon)$ be the solution of

$$
\delta_{k}+\varepsilon e^{\lambda_{k} t}=0
$$


that is,

$$
T(\varepsilon)=\frac{1}{\lambda_{r}} \log \left(-\frac{\delta_{k}}{\varepsilon}\right)
$$

where the sign of $\varepsilon$ is chosen such that $\delta_{k} / \varepsilon<0$.

Now let \|\| be the usual norm on $\mathbf{C}^{m}$. Since $S(t)$ is unitary, $P_{j}(t)$ are $\hat{W}\left(\lambda_{j}\right)$-polynomial functions and $a_{j}-\lambda_{r}<0(j=1, \cdots, r-1)$, we obtain in terms of (3.16) and (3.17), the following estimates of $\left\|d \tilde{z}_{k} / d t\right\|$ at $t=T(\varepsilon)$ for small $|\varepsilon|, 0<|\varepsilon|<\delta$ :

$$
\left\|\frac{d \tilde{z}_{k}(t ; \varepsilon)}{d t}\right\|_{t=T(\varepsilon)} \leqq K_{r}|\varepsilon|^{(1-\jmath r)}
$$

for an enough small positive number $\delta$, where $K_{r}$ is a constant independent of $\varepsilon$ and $f_{r}$ denotes $\max \left\{a_{1} / \lambda_{r}, \cdots, a_{r-1} / \lambda_{r}\right\}$.

We can now resime the above conclusions in the form of

THEOREM 3.6. Let $z(t)=S(t) \alpha$ be a $U(m)$-solution and $z(t ; \varepsilon)$ a solution with initial values $\alpha+\varepsilon \beta$. Suppose that there exists a simply dominant eigenvalue $\lambda_{r}$ for $\beta$ and that $\beta$ is $k$-dominant parallel to $\alpha$ with a real ratio-constant $\delta_{k},(1 \leqq h \leqq n)$. Then $\tilde{z}(t ; \varepsilon)$, the $\varepsilon$-order approximation of $z(t ; \varepsilon)$, has the estimate for small $|\varepsilon|$ :

$$
\left\|\frac{d \tilde{z}_{k}}{d t}\right\|_{t=T(\varepsilon)} \leqq K_{r}|\varepsilon|^{(1-f r)}
$$

where

$$
T(\varepsilon)=\frac{1}{\lambda_{r}} \log \left(-\frac{\delta_{k}}{\varepsilon}\right),
$$

and $K_{r}, f_{r}$ are constant as in (3.18) such that $f_{r}<1$.

In particular if $s=0$ and $r=1$, then

$$
\left.\frac{d \tilde{z}_{k}}{d t}\right|_{t=T(\varepsilon)}=0
$$

Remark. Suppose $\Gamma_{1} \Gamma_{2}+\Gamma_{2} \Gamma_{3}+\Gamma_{3} \Gamma_{1}<0$ in the equation (E). We take $\alpha_{1}=-1 / 2, \alpha_{2}=1 / 2, \alpha_{3}=\sqrt{-3}$ as initial values. Then $\hat{B}$ has eigenvalues $\lambda=\sqrt{-3\left(\Gamma_{1} \Gamma_{2}+\Gamma_{2} \Gamma_{3}+\Gamma_{3} \Gamma_{1}\right)},-\lambda, \pm 0$, and $\pm \sqrt{-1}\left(\Gamma_{1}+\Gamma_{2}+\Gamma_{3}\right)$. Take $\Gamma_{1}=-2$ and $\Gamma_{2}=1$. Then the eigenvector $\beta$ corresponding to the above simple-dominant root $\lambda$ is 1 -parallel to $\alpha=\left(\alpha_{1}, \alpha_{2} \alpha_{3}\right)$. It is sufficient 
to take $\Gamma_{3}=2$, a root of the equation $\sqrt{(X+2)}\left(X^{2}+4 X+4\right)-\left(2 X^{3}+\right.$ $9 X-2)=0$.

\section{2. $C^{*}$-solutions}

3.2.1. In this paragraph we treat an another kind of solutions.

Definition 3.7. Let $I$ be an open interval containing 0 . A solution $z(t)$ of (1.7) with $z(0)=\alpha$ is called a $\mathbf{C}^{*}$-solution if there is a smooth function $f: I \rightarrow \mathbf{C}^{*}$ such that

$$
z(t)=f(t) \alpha \quad(f(0)=1),
$$

where $\alpha=\left(\alpha_{1}, \cdots, \alpha_{n}\right) \in V$ and all vectors $\alpha_{j}$ are non-zeros.

Let $z(t)=f(t) \alpha$ be a $\mathbf{C}^{*}$-solution with initial conditions $z(0)=\alpha$. Then we have from (1.7) and (2.7)

$$
\bar{f} \dot{f} \alpha=\bar{X}(\alpha)
$$

where $\dot{f}$ means $d f / d t$. Therefore $\bar{f} \dot{f}$ being constant, we can set

$$
c=\vec{f} \dot{f}
$$

whence it follows

$$
c \alpha=\bar{X}(\alpha)
$$

Here putting $c=a+\sqrt{-1} b$, we find by (3.20)

$$
\frac{d}{d t}|f|^{2}=2 a \text {. }
$$

The solution $f(t)$ of this differential equation under the initial condition $f(0)=1$ is

$$
\left\{\begin{array}{l}
f(t)=\sqrt{2 a t+1} \exp \left\{\sqrt{-1} \frac{b}{2 a} \log (2 a t+1)\right\}, \\
|f|^{2}=2 a t+1 .
\end{array}\right.
$$

If $a=\operatorname{Re}(c)$ is zero, then the solution $z(t)$ reduces to $U(1)$-solution. On the other hand, if $a \neq 0$, then we can state the following

Proposition 3.8. The Hamiltonian function $H(z, \bar{z})$ is $\mathbf{C}^{*}$-invariant, i.e., the constant $\gamma$ in $(b)$ of $\S 1.2$ is zero. Moreover it follows

$$
\sum_{j=1}^{n} \Gamma_{j}\left\|\alpha_{j}\right\|^{2}=0
$$


Proof. At first it follows from (2.12) and (3.21) that

$$
\sqrt{-1} c \sum_{j=1}^{n} \Gamma_{j}\left\|\alpha_{j}\right\|^{2}=\gamma
$$

Since $\operatorname{Re}(c)=a$ is non-zero and $\gamma$ is real, we find $\gamma=0$, and so (3.23) is proved.

Now return to (3.21). Noting $\bar{f}(t) \bar{X}(f(t) \alpha)=c \alpha$, by (2.7) and (2.10)

$$
c \alpha+\bar{A}(\alpha) \bar{\alpha}=0 \text {. }
$$

Here as before let $\tilde{z}(t ; \varepsilon)=z(t)+\varepsilon f(t) v(t)$ be an $\varepsilon$-order approximation with initial values $\alpha+\varepsilon \beta$. To obtain differential equations which $v(t)$ satisfies, we take the independent variable $\tau$ as

$$
\frac{d}{d \tau}=|f|^{2} \frac{d}{d t}
$$

i.e.,

$$
\tau=\frac{1}{2 a} \log (2 a t+1)
$$

Then the system of differential equations for $v(\tau)$ is

$$
\frac{d v}{d \tau}=-c v(\tau)+\bar{A}(\alpha) \bar{v}(\tau)
$$

Similarly as (3.7) we define an $R$-linear map $B: V \rightarrow V$ by

$$
B(x)=-c x+\bar{A}(\alpha) \bar{x}
$$

for any $x \in V$, and so (3.26) can be written as

$$
\frac{d v}{d \tau}=B(v)
$$

Further we can write (3.28) in the real form

$$
\frac{d}{d \tau}\left(\begin{array}{l}
v_{1} \\
v_{2}
\end{array}\right)=\hat{B}\left(\begin{array}{l}
v_{1} \\
v_{2}
\end{array}\right)
$$

where $v=v_{1}+\sqrt{-1} v_{2}$ and $\hat{B}$ is the real matrix of $B$ on $V_{R}$. From Lemma 3.2 it follows that the characteristic equation of $\hat{B}$ is

$$
|(\lambda+c)(\lambda+\bar{c}) E-A \bar{A}|=0 .
$$


Thus we can prove the following

Proposition 3.9. ( i ) $-(c+\bar{c}), 0,-c$ and $-\bar{c}$ are eigenvalues of $\hat{B}$, and the vectors $c \alpha$ and $\sqrt{-1} \alpha$ are eigenvectors corresponding to $-(c+\bar{c})$ and 0 , respectively.

(ii) The matrix $A \bar{A}$ has eigenvalues 0 and $|c|^{2}$.

3.2.2. Let us return to the singularities of $\tilde{z}(t ; \varepsilon)$. Using $d / d \tau=$ $|f|^{2} d / d t$, we find from (3.26)

$$
\bar{f}(t) \frac{d \tilde{z}}{d t}=c \alpha+\varepsilon \bar{A}(\alpha) \bar{v}(\tau) .
$$

Assume the following conditions (F) are satisfied: (F) There is a simpledominant eigenvalue for $\beta$, say $\lambda$ and $\beta$ is $k$-dominant parallel to $\alpha$ with a real ratio-constant $\delta_{k}$. Then put

$$
T(\varepsilon)=\frac{1}{2 a}\left(\left(-\frac{\delta_{k}}{\varepsilon}\right)^{2 a / 2}-1\right)
$$

for $a=\operatorname{Re}(c)$. Then we can prove by the same procedures as 3.1 .2 the following

THEOREM 3.10. When the condition (F) is satisfied, the e-approximation $\tilde{z}(t ; \varepsilon)$ has the same estimates as (C) in Theorem 3.6 at $t=T(\varepsilon)$.

In particular, if there is only one eigenvalue $\lambda$ of $\hat{B}$ which is associated with $\beta$ and $s$ simply dominant, and if $\beta$ is $k$-dominant parallel to $\alpha$ with a real ratio-constant, $\delta_{k}$, then

$$
\left.\frac{d \tilde{z}_{k}}{d t}\right|_{t=T(\varepsilon)}=0
$$

We may conjecture that the constants $a, b$ in the problem (A) for the motion-equation (E) are given by the same relations $a=1 / \lambda_{r}, b=\left(\log -\delta_{k}\right) / \lambda_{r}$ appearing in $T(\varepsilon)$ in Theorem 3.6.

\section{REFERENCES}

[1] H. Lamb, Hydrodynamics, Cambridge (1932).

[2 ] J. L. Synge, On the motion of three vortices, Canad. J. Math., 1 (1949), 257-270.

[ 3 ] L. Pontrjagin, Ordinary Differential Equations, Kyoritsu (1964).

[ 4 ] S. Sternberg, Lectures on Differential Geometry, Prentice-Hall, New Jersey (1965). 
H. Omoto

Department of Mathematics

School of Science

Nagoya University

Chikusa-ku, Nagoya 464-01

Japan

Current address of $\mathrm{H}$. Omoto

Nagoya University College of

Medical Technology

Higashi-ku, Nagoya 461

Japan

Y. Kimura

The Institute for Solid State Physics

University of Tokyo

Minato-ku, Tokyo 106

Japan 